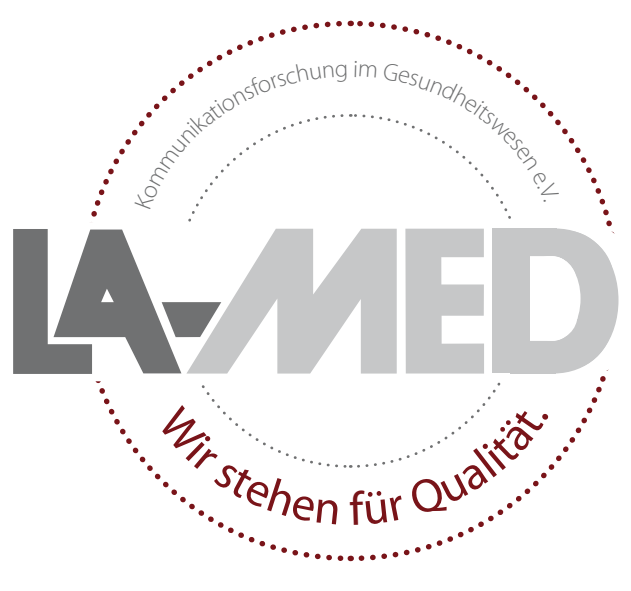

Sielesen
Qualität

\section{Damit das auch so bleibt,}

befragen wir Sie in

Kooperation mit tns infratest

in den nächsten Wochen.

\section{Ihr Urteil ist uns wichtig.}

Bitte nehmen Sie teil!

0
0
0
0
$\simeq$
$\frac{0}{0}$
$\frac{0}{\square}$
0

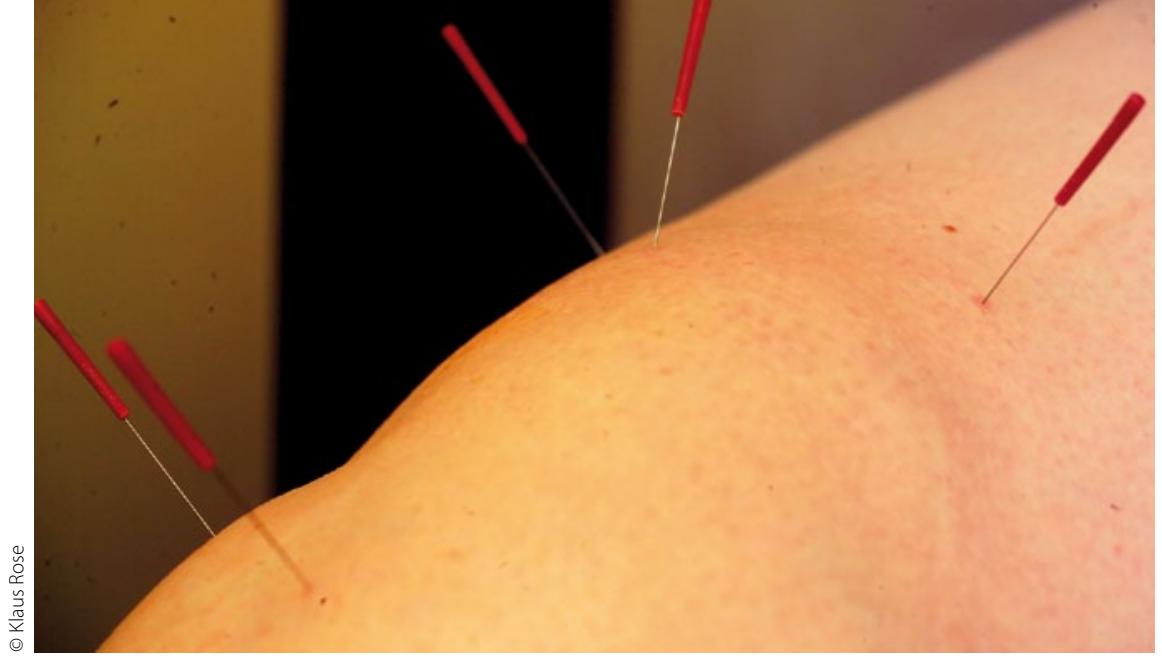

Akupunktur soll gegen die Fatigue der Krebspatienten wirksam sein.

\title{
Akupunktur gegen die Müdigkeit bei Krebspatienten?
}

\begin{abstract}
Krebsbedingte Müdigkeit ist ein häufiges, lästiges und nur schwer therapierbares Symptom. Viele Behandlungsformen werden empfohlen, aber nur sehr wenige sind gut belegt. Vor diesem Hintergrund wäre es schön, eine Therapie zu haben, die erwiesenermaßen hilft und nicht mit ernsten Nebenwirkungen belastet ist.
\end{abstract}

- Britische Kliniker randomisierten 302 Krebspatienten, die an mittel- bis schwergradiger, krebsbedingter Müdigkeit litten, in zwei Gruppen. Die Experimentalgruppe erhielt zusätzlich zur Standardbehandlung regelmäßig sechs Wochen lang Akupunktur, während die Kontrollgruppe nur die Standardtherapie verabreicht bekam. Zielparameter der Studie war die krebsbedingte Muedigkeit, die mit einem validierten Fragebogen erfasst wurde.

Bei Therapieende zeigte sich, dass dieses Symptom in der Experimentalgruppe signifikant weniger beklagt wurde. Dementsprechend war auch die Lebensqualität bei diesen Patienten signifikant höher. Die Autoren schließen aus diesen Daten, dass „Akupunktur eine effektive Intervention" bei dieser Indikation ist.

- A. Molassiotis et al.

Acupuncture for cancer-related fatigue in patients with breast cancer: a pragmatic randomized controlled trial. J. Clin. Oncol. 2012 Oct 29. [Epub ahead of print]

\section{Kommentar}

Die Autoren dieser Untersuchung scheinen der festen Überzeugung zu sein, dass sie eine Therapie für die krebsbedingte Müdigkeit identifiziert haben. Ich habe jedoch erhebliche Zweifel. Der Grund für mein Zögern liegt im Studiendesign; pragmatische Studien dieser Art können prinzipiell keinen Kausalzusammenhang zwischen der Therapie per se und dem klinischen Resultat herstellen. Es ist nämlich durchaus möglich, ich meine sogar wahrscheinlich, dass die Akupunktur mit einem erheblichen Placeboeffekt assoziiert ist. In diesem Fall mag die Akupunktur allein mittels unspezifischer Effekte das klinische Ergebnis bewirkt haben. Manche werden vielleicht meinen, dass das letztlich belanglos sei-Hautsache es hilft den häufig schwer leidenden Patienten. Auch da bin ich anderer Meinung: Falls das Result auf einem Placeboeffekt beruht, wäre es nicht richtig, Akupunktur im großen Stil in der klinischen Routine einzusetzen; dies wäre nicht nur recht kostspielig, es wäre auch ziemlich unsinnig - denn eine einfache, professionelle Zuwendung mit Empathie und Verständnis könnte wirksamer, nebenwirkungsärmer und billiger sein. Schwer leidende Patienten verdienen Besseres als ein Placebo, und wir wünschen uns aussagekräftigere Studien, um das Bessere zu finden.

E. ERNST - 